\title{
Les relations entre savoirs linguistiques et savoirs de spécialité dans la construction des processus de passage de l'être " cultivé » à l'être " civilisé »
}

\section{Françoise Raby}

\section{(2) OpenEdition}

\section{Journals}

Édition électronique

URL : http://journals.openedition.org/asp/4010

DOI : $10.4000 /$ asp.4010

ISSN : 2108-6354

Éditeur

Groupe d'étude et de recherche en anglais de spécialité

\section{Édition imprimée}

Date de publication : 1 décembre 1994

Pagination : 21-30

ISSN : 1246-8185

\section{Référence électronique}

Françoise Raby, «Les relations entre savoirs linguistiques et savoirs de spécialité dans la construction des processus de passage de l'être "cultivé » à l'être « civilisé » », ASp [En ligne], 5-6 | 1994, mis en ligne le 25 novembre 2013, consulté le 30 avril 2019. URL : http://journals.openedition.org/asp/4010 ; DOI : 10.4000/asp.4010

Ce document a été généré automatiquement le 30 avril 2019.

Tous droits réservés 


\title{
Les relations entre savoirs
} linguistiques et savoirs de spécialité dans la construction des processus de passage de l'être « cultivé » à l'être « civilisé »

\author{
Françoise Raby
}

1 Si les langues de spécialité ont conquis leur place dans le champ d'investigation de la linguistique, il semble plus difficile d'en dire autant d'un point de vue didactique, comme en témoignent les discussions animées qui ont eu lieu lors du dernier colloque RANACLES tenu au CNAM à Paris en décembre 1993.

2 C'est que cet enseignement se définit avant tout de manière institutionnelle, comme celui d'une langue étrangère aux spécialistes d'une autre discipline. On classera donc dans cette rubrique l'enseignement de l'anglais à des ingénieurs hydrauliciens, à des étudiants en arts plastiques, des techniciens de l'imprimerie, informaticiens, juristes, sociologues, futurs hôteliers, secrétaires, visiteurs médicaux... La liste semble illimitée et on se prend à penser qu'au fond l'enseignement de spécialité se définit surtout par ce qu'il n'est pas : l'enseignement des langues aux spécialistes de langues, un point c'est tout. La langue de spécialité serait donc une auberge espagnole où l'on parlerait essentiellement anglais.

3 Pourtant il est possible de mener une réflexion didactique sur ce type d'enseignement car au-delà de la diversité des contenus il présente des caractéristiques didactiques communes. L'une d'elles, commune à tout apprentissage, est la confrontation qui s'établit entre les connaissances nouvelles à acquérir et l'ensemble des croyances, des connaissances, qui préexistent sous forme d'images mentales, de représentations symboliques ou de raisonnement dans l'esprit de l'étudiant et qui constituent sa culture. L'objet de cette contribution est de réfléchir sur la manière dont s'organise cette confrontation entre langue et culture dans le cas particulier de la langue de spécialité. 
4 Nous avons vu que les langues de spécialité paraissent sans grande unité, autre qu'institutionnelle. Quant au mot culture, il se dresse d'emblée comme un second récif. Chargé d'histoire, envahissant tous les domaines du savoir, objet d'enjeux affectifs conscients ou inconscients, personnels ou collectifs, au fil des siècles il n'a cessé de rompre les mailles du filet sémantique. La confrontation entre les deux termes passera donc d'abord par une tentative taxinomique. Comme toute définition, celles qui seront proposées ici sont sujettes à contestation, mais elles ont pour objet, à travers l'éclaircissement terminologique de tenter de problématiser la relation entre cultures et langues de spécialité.

\section{Culture et civilisation}

5 La plupart des dictionnaires ne distinguent pas vraiment entre culture et civilisation. Pris dans leurs acceptions courantes ou scientifiques les deux termes sont d'une plasticité sémantique étonnante. Il paraît donc indispensable de définir de quoi on parle avant même de problématiser la relation centrale.

6 Nous définirons la culture comme l'ensemble des croyances, connaissances et savoirs qui fait qu'un individu se reconnaît appartenir à une communauté. La culture est donc caractérisée par le lien et la clôture. La croyance est de l'ordre de l'adhésion, il faut y ranger les mythes, les pratiques religieuses et sociales, les rites.

7 Si quelqu'un prétend détenir un savoir, il doit pouvoir à chaque instant apporter la preuve de ce qu'il avance. Les savoirs, au sens strict, supposent donc, entre autres, la falsifiabilité à travers l'expérimentation (Popper). Ils définissent la pensée scientifique.

Les connaissances se situent à mi-chemin entre savoirs et croyances. Elles sont de l'ordre de la croyance en ce qu'elles relèvent d'un acte d'adhésion, elles sont de l'ordre du savoir en ce qu'elles reposent sur des savoirs produits par des experts. Si on retient cette distinction, on s'accordera à constater que l'École ou l'Université dispense beaucoup plus de connaissances que de savoirs.

9 Du côté de la culture, on trouve la communauté : religieuse, sportive, sociale, politique, etc. La communauté organise son activité, son rapport au monde selon un modèle commun, mais non thématisé. La communauté ne prend pas sa culture pour objet de réflexion, elle se contente de « l'expérencier ». La culture est anhistorique.

10 Le processus de civilisation peut être envisagé au niveau de l'histoire des sociétés comme au niveau de l'histoire de l'individu. Sur le plan de l'histoire des sociétés, il indique le processus par lequel la circulation des hommes amène la circulation des idées. La circulation des idées c'est la confrontation des croyances et des connaissances, la confrontation des cultures. De cette confrontation naissent de nouveaux savoirs, de nouvelles croyances, de nouveaux modèles culturels. La circulation des hommes et des idées provoque la prise de conscience de l'identité culturelle. La communauté se prend elle-même pour objet de réflexion : sa langue, sa religion, sa culture, etc. Elle thématise les traits qui la distinguent d'autres communautés. La civilisation, c'est l'émergence de l'histoire.

11 Mais le processus qui mène de la culture à la civilisation ne suit pas forcément un ordre séquentiel : par exemple, au mythe, à la croyance succéderait l'échange, la circulation, puis la civilisation : le logos. Il s'agit en réalité d'une relation dialectique au sens hégélien du terme: une marche de la pensée qui consiste essentiellement à reconnaître 
l'inséparabilité des contradictoires. Le passage de la culture à la civilisation exige cette inséparabilité (Einheit) des contradictoires : il faut bien qu'existe d'abord les mondes clos des cultures communautaires pour que s'instaurent l'échange et les mondes civilisés.

L'histoire de la linguistique nous offre, par ailleurs, un exemple très intéressant de ce passage. Auroux signale en effet qu'en Grèce les premières tentatives d'analyse linguistique initiées par les sophistes et les rhéteurs sont antérieures aux mythes du Nomothète, d'Hermès ou de Thot. On a donc là production d'un mythe après la production de savoirs. Les deux vont d'ailleurs coexister. Et cela s'explique par le fait que dans les civilisations anciennes les premières analyses linguistiques sont des entreprises closes et monolingues il y a production d'un certain savoir sans qu'il y ait eu pour autant échange. Il faut attendre la Renaissance pour voir les Occidentaux se lancer dans la description des langues du monde à partir des catégories grecques et occidentales. On ne peut comprendre cette immense entreprise, souvent occultée par les progrès scientifiques de l'époque dans le domaine des mathématiques et des sciences de la nature, sans la découverte de l'imprimerie, sans le développement des vernaculaires européens, sans les découvertes et les voyages.

\section{L'apprentissage}

La culture d'un individu se définit avant tout par sa langue, laquelle manifeste son rapport au monde et aux autres hommes. La langue traduit une macro-culture. Pour désigner cette macro-culture nous reprendrons, faute d'une meilleure formulation, l'expression «culture communautaire » (Zarate 1986). Mais tout individu, à mesure qu'il se développe, construit des micro-cultures qui correspondent à tel ou tel champ de sa pratique sociale : culture sportive, culture culinaire, culture politique. Ce développement reproduit, à travers le processus de socialisation, l'histoire du passage de la culture à la civilisation.

L'école est le lieu par excellence du passage qui ne se fait pas sans heurt, car tout apprentissage suppose un exil, une mue. Michel Serres (1991) choisit l'image du manteau d'Arlequin pour représenter le phénomène chaotique, non linéaire, aléatoire de l'apprentissage : revêtu du manteau bigarré d'Arlequin, le maître apparaît dans la classe. « Puissante et plate, la parole règne, monotone, elle vitrifie l'espace.... » Mais le manteau du maitre contredit sa parole: " ... superbe de misère, le vêtement éblouit.» Car le vêtement raconte le voyage du maître :

Bigarrure composite, faite de morceaux en haillons ou lambeaux, de toutes tailles, mille formes et couleurs variées, d'âges divers, de provenances différentes, mal faufilés, juxtaposés sans harmonie, sans attention portée aux voisinages, reprisés selon les circonstances, à mesure des besoins, d'accidents et de contingences, montre-t-elle une sorte de mappemonde, la carte des voyages du comédien, comme une valise constellée de marques?

Ainsi, le manteau du pédagogue prévient-il silencieusement l'enfant, tel la pythie :

Aucun apprentissage n'évite le voyage. Sous la conduite d'un guide, l'éducation pousse à l'extérieur (....) Tes idées initiales ne répètent que des mots anciens. Jeunes : vieux perroquets. Le voyage des enfants, voilà le sens nu du mot pédagogie.

Dans la littérature, à partir du $18^{\mathrm{e}}$ siècle, les Bildungsroman nous figurent l'éducation et l'apprentissage comme un voyage, comme une mue : la perte de l'innocence. De Goethe à Doris Lessing, en passant par Fielding, Diderot, Dickens, ou plus récemment Joyce et 
Thomas Mann, le jeune héros construit sa personnalité à travers de multiples péripéties. Chacune d'entre elles contient une épreuve initiatique qui lui arrache un pan d'innocence, tandis que se tisse, parfois malgré lui, la seconde peau de la sagesse.

L'école, dans son sens large, est le lieu étrange où l'on voyage immobile. Et ce voyage conduit au sentiment d'exil ou de liberté (Jacques Baillé) :

Partout où la formation s'éprouve comme médiation vers une vie matérielle et spirituelle espérée meilleure, vers une vie dont le présent ne signale que l'absence, cette même formation s'éprouve aussi comme carence de la vie, comme exil, comme aliénation (au sens exact de l'étymologie). Partout où la formation s'éprouve comme plénitude d'un présent absorbé dans l'heureuse exécution d'une tâche, comme l'immédiate gratification spéculaire délivrée par la conscience réfléchissant ses propres conquêtes, cette même formation s'éprouve aussi comme liberté.

En accédant à l'Université l'étudiant achève le premier voyage de la formation générale, pour accéder à celui qui devrait le conduire vers la vie professionnelle. Si tout se passe bien, les chemins qu'il parcourt à l'Université feront de lui un expert de et dans sa spécialité. Mais l'étudiant d'une langue étrangère de spécialité effectue en permanence un double voyage : sujet de culture, il confronte la culture communautaire, nationale, qu'exprime sa langue à la culture que véhicule la langue cible ; sujet rationnel, détenteur de savoir, il doit soumettre cet apprentissage au canal étroit et précis de la spécialité, qui exige d'autres abandons, d'autres mues. Bachelard n'évoquait pas autre chose lorsqu'il préconisait une lutte obstinée contre l'opinion, lorsqu'il affirmait que "toute culture scientifique doit commencer par une catharsis intellectuelle et affective».

C'est l'objet de la didactique de s'interroger sur la nature du voyage. C'est l'objet de la pédagogie de tenter d'intervenir pour en améliorer les conditions. L'exposé qui suit se situe modestement dans l'optique de la première. Il vise à décrire et comprendre comment les divers éléments culturels, linguistiques et disciplinaires interagissent dans la production/ou l'absence de production de nouvelles connaissances linguistiques et/ou disciplinaires. Mais pour tenter de problématiser ces interactions, il convient d'abord de classer les discours de spécialité.

\section{Les discours de spécialités}

\section{Langues naturelles/langues formelles}

Un document de spécialité mélange souvent différents langages: langages naturels, langages formels. À l'intérieur des langages naturels il suppose la confrontation langue maternelle/langue cible. À l'intérieur des langages formels, il fait appel à des catégories sémiotiques : icônes, graphes, tableaux, diagrammes, etc. On voit bien que comprendre un texte de spécialité suppose une maîtrise de plusieurs codes et langages, la langue étrangère ajoutant un nouveau système sémiotique.

21 L'étudiant d'économie, de maths, de physique, de communication, a rarement affaire uniquement à la langue naturelle. Une partie du contenu du texte peut être représenté sous forme de graphes, de schéma, de diagrammes, de tableaux, d'images, etc. Cognitivement, cela pose un certain nombre de problèmes qui n'ont semble-t-il encore guère été abordés dans le cas des L2. Il s'agit du passage d'un registre sémiotique à un autre. 
Exemple: L'étude d'un document portant sur l'évolution du chômage aux USA. Pour comprendre le document, l'étudiant devra s'appuyer sur trois types de connaissances : la connaissance de l'anglais de base, des connaissances suffisantes sur l'économie américaine pour contextualiser correctement le document, mais également, dans le cas où certains énoncés sont remplacés par des graphes, un savoir-faire : la maitrise de ce registre sémiotique particulier. L'interprétation correcte des graphes indiquera son niveau général d'expertise dans la spécialité, mais la question de savoir pourquoi il a atteint ou non le niveau requis peut éventuellement s'expliquer culturellement, tel ou tel registre sémiotique intervenant plus souvent dans les textes d'économie d'un pays à l'autre.

Certains enseignements, comme les mathématiques, utilisent largement la langue comme un code. Il s'agit d'un langage univoque, de l'ordre de la dénotation. Le discours fait peu appel à la langue générale. Du point de vue didactique, c'est un enseignement très fortement marqué par le niveau de connaissances dans la spécialité, et qui stimule des modèles mentaux liés essentiellement à la faculté rationnelle. Il convient toutefois de signaler qu'univocité ne signifie pas pour autant « transparence » entre les langues (Petit 1993).

\section{Discours scientifique, discours technique, discours rhétorique}

Pour qu'il y ait acte didactique, c'est-à-dire transmission de connaissances, il faut qu'il y ait eu accord sur le sens du document étudié et accord sur le critère de vérité. Le discours scientifique, discours formel et logique, égrène ses tables de vérité, à mesure qu'il se développe. Il ne devrait donc pas poser de problème particulier à l'étudiant de langue étrangère. En réalité l'étude des articles scientifiques soulève de nombreuses questions d'ordre culturel (cf. les articles de la revue ASp 2 sur « contraintes et libertés »).

Bruno Latour (1988), pour sa part, a démontré que parfois le discours scientifique se ramène en réalité à un discours rhétorique, et qu'il cache un combat économique sans merci pour occuper le champ scientifique, les règles et l'issue du combat n'étant perceptibles que pour les initiés.

Mortureux et Petit (1986) attirent notre attention sur les discours scientifiques de vulgarisation :

Les discours de vulgarisation apparaissent «seconds " par rapport aux discours scientifiques proprement dits. Ils se caractérisent comme discours « rapportant», «traduisant", par rapport à des discours primaires. Ils créent de véritables paradigmes désignationnels et définitionnels, réseaux de vocables fonctionnant en co-référence avec un vocable scientifique donné.

Le discours vulgarisant renvoie chez l'étudiant non plus aux connaissances ou aux savoirs de spécialité, mais à la culture de spécialité : ensemble des croyances qui se mêlent aux savoirs dont il dispose dans le domaine de la spécialité. Le document en langue étrangère se trouve alors véhiculer une double culture : la culture communautaire et la culture de spécialité. La compréhension du document dépend de la manière dont est structuré le double transfert didactique : langue étrangère et spécialité.

Le discours technique ne se donne pas comme «représentation» du monde mais directement comme construction du monde, comme moyen d'action sur la réalité. Il porte sur le savoir-faire, et le critère de vérité est dans la réalisation de la tâche. L'acquisition de l'anglais du téléphone, ou de l'anglais de l'informatique ne devrait donc 
pas poser de gros problèmes. En réalité, du point de vue du lexique comme du point de vue syntaxique, peut-être est-ce dans cet anglais-là, le plus pragmatique, donc elliptique et idiomatique, que se révèlent les plus gros obstacles culturels (phrasal verbs, constructions terminologiques de spécialité). Peut-être parce que la langue technique sécrète non seulement son propre lexique mais plus encore une grammaire spécifique.

Le discours des sciences humaines, texte journalistique, commentaire historique, en gros, la langue des sciences molles pose des problèmes didactiques également délicats. D’abord parce que les thèmes abordés couvrent des champs d'activité et de connaissances extrêmement larges. D'autre part parce qu'on se situe clairement dans le domaine linguistique de la connotation (Mounin 1963). La charge sémantique du document est alors très importante Il s'agit, pour l'étudiant, de déjouer les pièges d'un lexique dont le sens varie continuellement (ex. le concept de "travail»), voire d'une époque à l'autre, d'une discipline à l'autre : droit, économie, d'une école à l'autre, etc. De plus, il convient aussi de repérer les figures du discours propres à chaque discipline.

La question qui se pose alors, en classe, est celle du critère de vérité. Dans le domaine des faits, comme dans le domaine des jugements, quelle situation didactique mettre en place pour qu'à travers la langue étrangère de spécialité s'organise l'échange culturel entre les étudiants, l'enseignant et le document étudié, de sorte que la question du sens du document soit l'occasion pour l'étudiant de thématiser sa propre culture autant que de comprendre la culture étrangère.

\section{Quelques problèmes d'ordre didactique}

Devant une telle variété dans les discours, peut-on encore prétendre parler de « langue de spécialité » d'un point de vue didactique ? C'est à dire peut-on passer de la diversité des types de discours à l'unicité ou l'universalité d'une catégorie didactique définie par un singulier? En d'autres termes peut-on repérer quelques traits didactiques propres à la langue de spécialité?

D'abord l'enseignement de langue étrangère de spécialité répond à des objectifs utilitaires. Il s'agit de permettre aux étudiants de s'approprier la langue nécessaire à la pratique de spécialité. C'est pourquoi la langue de spécialité se définit avant tout comme une langue outil. Il s'agit donc de sélectionner dans le champ immense des possibles offerts par la langue-objet les outils sémantiques et syntaxiques nécessaires à la spécialité.

33 Sélectionner d'abord dans l'anglais. L'anglais sans épithète, ni de communication, ni de spécialité, ni professionnel, tellement évident, tellement nécessaire qu'on ne lui a toujours pas trouvé de meilleur label qu' «English for general purposes ». Cet anglais de base est le fond commun de la langue, dans lequel tout spécialiste doit puiser pour construire son discours de spécialité. L'apprentissage de cet anglais pose en gros le même type de problème didactique, quelle que soit la spécialité. Cet anglais, si limité soit-il, confronte l'étudiant à une vision du monde (Sapir, Whorf, Mounin) différente de celle dont est porteuse sa propre langue. Le problème peut se poser au niveau syntaxique le plus simple. Ex. : comment l'étudiant reconnaît-il le sujet dans une phrase ? Est-ce à travers l'ordre des mots, le cas, l'accord, la différence animé inanimé (Harrington 1987) ? Mais il se pose aussi de manière cruciale sur le plan sémantique, avec notamment la richesse métaphorique de la langue de base, qu'il s'agisse de métaphore morte ou de métaphore 
vive. La compréhension et l'appropriation de ces métaphores supposant de la part de l'étudiant la prise de conscience de modèles mentaux différents, à l'œuvre dans sa propre langue ou dans la langue cible. Il s'agit bien là d'un problème d'ordre culturel (Lakoff \& Johnson 1985).

Il convient ensuite de sélectionner dans la langue de spécialité et de mettre en forme le discours de spécialité qui servira de base à la situation didactique. Le discours de spécialité s'inscrit dans une perspective résolument pragmatique, au sens banal du terme comme au sens savant. Au sens banal du terme, nous en avons souligné l'aspect utilitaire ; au sens savant, puisque l'acte didactique implique la contextualisation des énoncés dans une situation de communication mettant en relation enseignant et enseignés. De plus le critère d'acquisition d'une langue étrangère de spécialité ne se mesure pas tant à l'aulne de prestations grammaticales ou métalinguistiques, qu'à la capacité pour l'étudiant de réaliser des tâches propres à la spécialité. C'est très clairement la forme d'enseignement que l'anglais dit goal-driven, donc pragmatique (cf. la création du DNCL).

Cette double activité de sélection de la part de l'enseignant pose le problème de la situation didactique en terme d'expertise. Il faudrait pouvoir modéliser l'apprentissage de la langue étrangère de spécialité à travers la relation savoir linguistique/savoir disciplinaire ; culture communautaire maternelle/culture communautaire cible,/culture de spécialité, etc. Impossible d'envisager un tel projet holistique dans l'état actuel des connaissances sur l'apprentissage et la cognition. Une telle formalisation supposerait que soient tranchés les débats qui opposent entre eux les cogniticiens au sujet du mystère de la boîte noire.

Cependant, si elles ne nous ont pas encore livré la "vérité ", les sciences cognitives offrent aux didacticiens des modèles concurrents, qui ont au moins le mérite de porter un regard nouveau sur l'apprentissage. Impossible, dans le cadre limité de cette contribution, de passer en revue un grand nombre de modèles. Nous avons choisi de faire référence aux derniers travaux de Noam Chomsky, en montrant, le cas échéant, comment il s'oppose à d'autres modèles.

37 S'agissant de Chomsky il conviendrait, d'ailleurs, de parler de théorie puisque de modèle en modèle, au fil des années, la pensée de Chomsky, héritière de Descartes et de PortRoyal, s'est constituée en véritable théorie. Cette théorie ne nous fournit pas directement de modèle de problématisation puisqu'elle s'intéresse clairement à la compétence, c'est-àdire à une potentialité, définie génétiquement et qui s'exprime dans les diverses facultés de l'esprit. Cependant, nous allons voir que dans l'article présenté ici, «Mental constructions and social realities » (1993), les problèmes posés rejoignent directement nos préoccupations.

De manière assez provocatrice et sibylline, Chomsky commence ainsi son exposé : « Il y a le problème de Platon et il $\mathrm{y}$ a le problème d'Orwell ». Le problème de Platon c'est: comment pouvons-nous savoir et comprendre tant de choses, étant donnée la pauvreté de notre environnement? Le problème d'Orwell c'est: comment pouvons-nous savoir et comprendre si peu de choses, étant donnée la richesse de notre environnement?

Nous voici, d'emblée, au cœur de notre problème : comment mes étudiants peuvent-ils savoir et comprendre autant d'anglais étant donné la pauvreté d'exposition à la langue : une heure par semaine, trente étudiants par groupe; ou bien, comment mes étudiants peuvent-ils savoir et comprendre si peu d'anglais, étant donné la richesse de l'environnement linguistique anglophone dans lequel ils évoluent? 

structures cognitives collaborent, c'est à dire comment l'information est computationnée d'un système à l'autre. Il est encore trop tôt pour savoir si les théories connexionistes répondront à ces questions, mais on trouve déjà des éléments de réponses dans les théories du traitement de l'information, dans les expérimentations sur les stratégies spécifiques de transferts linguistiques comme source de l'interlangue (cf. Harrington 1987). Chomsky, lui, se contente de formuler des hypothèses sur l'organisation du cerveau en systèmes et structures cognitives, en proclamant qu'on ne peut guère aller plus loin que l'abduction, puisqu'il n'existe pas, selon lui, de théorie satisfaisante de l'apprentissage. De ce point de vue, il rejette avec une égale vigueur tous les mécanismes 
généraux d'apprentissage appliqués à n'importe quel domaine : modèles connexionistes, traitement de l'information, modèles compétitifs, modèle d'Anderson, etc.

Les soi-disant mécanismes d'apprentissage ne peuvent rendre compte », écrit-il, "de tâches aussi disparates que l'acquisition du langage, percevoir des objets en mouvement, ou comprendre la physique quantique » (Chomsky $1993: 35$ ).

En ce qui concerne le problème de la culture communautaire et sociologique, Chomsky l'aborde fondamentalement en terme de constructions mentales sociales. En effet, à la question que nous pourrions lui poser de savoir comment les images mentales, symboles, représentions se construisent peu à peu dans le cerveau, jusqu'à produire de véritables modèles mentaux, il répond par « une faculté de l'esprit affectée au domaine social qui nous permet de fonctionner avec le monde. " Voilà donc une troisième faculté mise à l'œuvre, dans l'apprentissage des langues étrangères de spécialité.

Une question tout à fait triviale vient alors à l'esprit: si le cerveau développe normalement toutes ces facultés, tels des organes, comment expliquer la possibilité d'échec de l'apprentissage? Chomsky répond indirectement à cette question de deux manières: il invoque le facteur externe et le facteur interne. Le facteur externe le conduit, semble-t-il (une fois n'est pas coutume), à faire une concession au behaviorisme : le problème se situe du côté de l'environnement. L'environnement pose des problèmes à l'apprenant que son cerveau ne peut traiter. Mais on s'aperçoit qu'on revient, bien vite, au facteur interne comme facteur déterminant: car si le cerveau est en échec c'est soit parce que les problèmes sont mal posés, donc que notre faculté d'abduction a mal fonctionné, soit que, de toute manière, ces problèmes échappent aux limites biologiques de notre intelligence. Plus subtilement encore, Chomsky suggère que notre faculté de langage, tout comme notre faculté d'abduction, nous permettrait de poser des problèmes dont elle saurait qu'elle n'a pas les moyens de les résoudre. En d'autres termes notre cerveau est capable de penser ses propres limites :

Il n'y a pas d'incohérence dans le fait de penser que notre faculté rationnelle peut ellemême nous fournir des éléments de connaissance sur son propre champ et ses propres limites.

Enfin, Chomsky définit la science comme la " convergence entre les structures cognitives et les propriétés du monde » (1993: 33). Une convergence immédiatement corrigée en faveur des structures cognitives, puisqu'il pense, avec l'appui de Goodman, que «les mondes sont plus faits que trouvés ".

Les thèses que développe Chomsky posent un certain nombre de questions concernant l'apprentissage d'une langue étrangère de spécialité qui permettront peut-être d'ouvrir une discussion :

- L'acquisition d'une langue étrangère relève-t-elle d'un problème ou d'un mystère? Ou de l'un et de l'autre, selon les individus, et à quelles conditions ?

- Si la faculté de langage est universelle, la capacité à apprendre une langue, elle, ne le serait pas?

- Comment expliquer ce qui entrave le fonctionnement cognitif : faculté de langage? faculté rationnelle? faculté sociale?

Mais pour revenir plus directement aux obstacles didactiques propres à l'enseignement d'une langue de spécialité, l'obstacle le plus important n'est pas forcément celui que l'on croit. Dans l'acquisition de la langue étrangère de spécialité, l'obstacle, ce n'est pas la spécialité, mais la langue. Et si c'est la langue, ce n'est peut-être ni la langue étrangère, ni 
la langue maternelle, ni la langue de spécialité, maternelle ou langue cible, mais quelque chose qui se situe plus en amont, entre la faculté de langage et la langue, non structuré cognitivement sous forme de modèles, ou de représentations : amorces de raisonnement, images partielles, embryons de modèles. Car c'est peut-être à ce niveau-là d'activité mentale, incomplet et labile, que l'information circule, pour un temps, de la langue source à langue cible, de la spécialité à la grammaire, pour finalement s'engager franchement dans le domaine linguistique ou le domaine de spécialité. Pendant une phase préliminaire, langue étrangère et spécialité échangent leurs statuts: parfois c'est la spécialité qui est le but et la langue joue le rôle d'outil, parfois c'est la spécialité qui est l'outil et la langue devient le but. Mais que dire alors, de « la frontière du mystère »?

\section{Babel revisité}

Si la faculté d'abduction échoue sur des mystères, la faculté de langage aura recours au mythe, à la malédiction, pour expliquer leur commun échec. Au commencement était la culture : ce monde clos du jardin d'Eden où Dieu fit don de la parole à Adam, pour qu'il sortît de l'animalité, et d'une compagne pour qu'il pût lui parler. Au commencement était la parole commune, monogénique, qui permettait aux descendants de Noé de se parler, de se comprendre de constituer un peuple et une culture. Mais un jour, lassés de voyager, et craignant de devoir se disperser, ces descendants décidèrent de bâtir une ville et de s'y installer. " Allons, bâtissons-nous une ville et une tour dont le sommet pénètre les cieux ! Faisons-nous un nom et ne soyons pas dispersés sur toute la terre. » Et voilà que la colère de Yahvé s'abat sur eux : « Voici que tous font un seul peuple et parlent une seule langue, et tel est le début de leur entreprise ! Maintenant aucun dessein ne sera irréalisable pour eux. Allons ! Descendons, et là confondons leur langage pour qu'ils ne s'entendent plus les uns les autres ». Le mythe reprend le thème de la chute, car il s'agit encore de punir les hommes par l'exil et la diaspora. Mais s'il s'agit encore du péché d'orgueil, il ne s'agit plus de vouloir goûter à l'arbre de la connaissance, mais de partager des connaissances érigées en savoir. La tour symbolise donc un nouveau défi : celui de la libération de l'homme par l'édification d'un savoir collectif : savoir technique, savoir savant, savoir de spécialité. Le mythe raconte en effet, comment les hommes, grâce à leur langue commune, collaborent à la construction de l'édifice. Contrairement à ce qu'avancent certains exégètes, ce n'est par la parole commune, qui suscite le courroux divin - comment en serait-il ainsi puisque cette parole fut don de Dieu? - mais bien par ce qu'ils en font. La tour de Babel, outre la malédiction des langues, nous raconte peut-être le mythe de la naissance douloureuse de la civilisation : construction de la première ville, construction du premier objet technique, partage des savoirs mais aussi des savoir-faire, langues de spécialistes, langues de spécialité.

\footnotetext{
Alors ils ont cherché des pauvres

Pour bâtir cette immense ville, Alors, dans l'hiver et l'été, ils ont rassemblé des manœuvres, des cimentiers et des maçons, des forgerons couleur de cuir et de suie, et des charpentiers, de noirs bûcherons aux mains vertes, des hommes aux bouches ouvertes comme des pélicans de mer.
} 
Soudain, ceux qui portaient les briques, les poutres, les rocs, tous ceux qui pétrissaient le sable et la chaux, ont agité leur langue lourde. Et la colère et la douleur et la musique de leurs mots fleurissaient aux murailles sourdes. Et chacun soudain reconnut le sens de ces corolles nues. Espoir et désir et lumière. (Gamarra1991)

\section{BIBLIOGRAPHIE}

Anderson, J. R. 1983. The Architecture of Cognition. Cambridge, MA : Harvard University Press.

Auroux, S. 1993. «Comment surmonter Babel ?». Monogénitisme et universalité linguistique.

Bachelard, G. 1972. La formation de l'esprit scientifique. rééd. Paris : Vrin.

Chomsky, N. 1993. « Mental constructions and social realities ». In Knowledge and Language vol. 1. Dordrecht, Boston, London : Kluwer Academic Publisher, 29-56.

Fodor, J. 1983. The Modularity of Mind. Cambridge, MA : The Massachussetts Institute of Technology Press.

Gamarra, Pierre. 1991. «Dizains de la multiplication des langues ». In Corps écrit 36.

Harrington, M. 1987. « Processing transfer: Language specific processing strategies as a source of interlanguage variation ». Applied Linguistics 8, 351-377.

Lakoff, M. et M. Johnson. 1985 [1980]. Les métaphores dans la vie quotidienne. Paris : les Éditions de Minuit, 1985. $1^{\text {re }}$ éd. « Conceptual metaphors in everyday language », Journal of Philosophy 77, 8.

Latour, B. et P. Fabri. 1977. « La rhétorique de la science, pourvoir et devoir dans un article de science exacte ». Actes de la recherche en sciences sociales 13, 81-95.

Mortureux, M. F. et G. Petit. 1986. « Fonctionnement du vocabulaire dans la vulgarisation et problèmes de lexique ». DRALV 40, 41.

Mounin, G. 1963. Les problèmes théoriques de la traduction. Paris : N. R. F. Gallimard.

Petit, M. 1993. «Contraintes et libertés dans le passage à l'anglais du discours mathématique ». ASp 2, 94-106.

Sapir, E. 1967. Anthropologie. Paris : Édition de Minuit.

Serres, M. 1991. Le Tiers instruit. Paris : François Bourin.

Whorf, B. L. 1956. Language, Thought and Reality. Cambridge, MA : The Massachussetts Institute of Technology Press.

Zarate, G. 1986. Enseigner une culture étrangère. Paris : Hachette, 1986. 


\section{RÉSUMÉS}

Où l'auteur esquisse quelques définitions des mots culture, civilisation et apprentissage et se demande s'il n'entraîne pas son ami lecteur dans une impasse. Où l'auteur tente de s'y retrouver dans les discours de spécialité et leur rapport à la culture et commence à désespérer. Où l'auteur se demande si les sciences cognitives pourraient lui fournir quelques modèles. Où il apparaît que les sciences cognitives sont en guerre et n'en disent pas bien long sur la culture. Épilogue : Où l'auteur, lassé du cognitif, quitte l'ordre des discours et des modèles pour trouver refuge dans la tour de Babel, et là, plagiant Sterne, laisse à son lecteur le soin de remplir la page vierge.

In which the author tentatively sketches a few definitions of words such as culture, civilisation and learning and wonders if she is not misleading her readers into a blind alley. In which the author desperately tries to clarify the relationship between "special purpose discourses", "culture" and "foreign language learning", eventually asking cogniticians for their help. In which the author wonders if, by any chance, the cognitive sciences might provide some models, and subsequently discovers that the cognitive sciences are at war with one another and, besides, do not seem to say much about "culture". Epilogue: in which the author, tired of cognition, leaves the world of logos and of scientific models to take refuge into that of myths, into the Tower of Babel, and there, plagiarizing Sterne, leaves it to you, reader, to fill the blank page.

\section{INDEX}

Keywords : civilisation, culture, language for specific purposes, learning, model

Mots-clés : apprentissage, culture, civilisation, langue de spécialité, modèle

\section{AUTEUR}

\section{FRANÇOISE RABY}

Françoise Raby enseigne à l'Université Pierre Mendes France-Grenoble 2 et est membre du Laboratoire des Sciences de l'Éducation/Centre des Langues Vivantes. fanchon.raby@gmail.com 